\title{
FUZZY O-CLOSURE OPERATOR ON FUZZY TOPOLOGICAL SPACES
}

\author{
M.N. MUKHERJEE \\ and \\ S.P. SINHA \\ Department of Pure Mathematics \\ University of Calcutta \\ 35, Ballygunge Circular Road \\ Calcutta - 700 019, INDIA \\ (Received March 1, 1988 and in revised form August 16, 1990)
}

\begin{abstract}
The paper contains a study of fuzzy $\Theta$-closure operator, $\Theta$-closures of fuzzy sets in a fuzzy topological space are characterized and some of their properties along with their relation with fuzzy $\delta$-closures are investigated. As applications of these concepts, certain functions as well as some spaces satisfying certain fuzzy separation axioms are characterized in terms of fuzzy $\Theta$ closures and $\delta$-closures.
\end{abstract}

KEY WORDS AND PHRASES. Fuzzy $\Theta$-cluster point, fuzzy $\Theta$-closure, fuzzy $\delta$-closure, q-coincidence, q-neighbourhood.

1980 AMS SUBJECT CLASSIFICATION CODE. Primary 54A40; Secondary 54C99, 54D99.

\section{INTRODUCTION.}

It is well-known that the concepts of $\Theta$-closure and $\delta$-closure, are useful tools in standard topology in the study of H-closed spaces, Katetov's and H-closed extensions, generalizations of Stone-Weierstrass' theorem etc. For basic results and some applications of $\Theta$-closure and $\delta$-closure operators we refer to Veličko [1], Dickman and Porter [2], Espelie and Joseph [3] and Sivaraj [4]. Due to varied applicabilities of these operators in formulating various important set-topological concepts, it is natural to try for their extensions to fuzzy topological spaces. With this motivation in mind the concept of $\Theta$-closure operator in a fuzzy topological space (due to Chang [5]) was introduced by us in [6] in the light of the notions of quasi-coincidence and q-neighbourhoods of $\mathrm{Pu}$ and Liu $[7,8]$. In the present paper our aim is to continue the same study which ultimately shows that different fuzzy topological concepts can effectively be characterized in terms of fuzzy $\Theta$-closure and $\delta$-closure operators.

In Section 2 of this paper we develop the concept of fuzzy $\Theta$-closure operators and characterize fuzzy $\Theta$-closures of fuzzy sets in a fuzzy topological space in different ways. In literature there can be found several definitions of $T_{2}$-spaces in fuzzy setting. We take the definition of fuzzy $T_{2}$-space as given by Ganguly and Saha [9] and become able to successfully characterize it in our context. Fuzzy regularity has been introduced by many workers from different view points, including one by us in [6]. Since our fuzzy regularity along with the fuzzy $T_{1}$-axiom (of [9]) does not yield the above fuzzy $T_{2}$-axiom, we propose to call it "strong $T_{2}$ " in fuzzy setting. Fuzzy semiregularity and almost 
regularity were also defined in [6]. We characterize fuzzy regularity and these weaker forms of fuzzy regularity in terms of fuzzy $\Theta$-closure and $\delta$-closure. All these characterizations are incorporated in Section 3 of the paper. Fuzzy weakly continuous functions were first introduced by Azad [10] and were further investigated in [11], whereas the concept of fuzzy $\Theta$-continuous functions was initiated in [6]. Section 3 also includes the characterizations of these functions with the help of the notion of fuzzy $\Theta$-closures.

We now recall some definitions and results of a fuzzy topological space (henceforth fts, for short) $(X, T)$ to be used in this paper excepting very standard ones for which we refer to Zadeh [12], Chang [5] and $\mathrm{Pu}$ and Liu [7,8]. The interior and closure of a fuzzy set $\mathrm{A}$ in an fts $(X, T)$ will be denoted by Int $\mathrm{A}$ and $\mathrm{Cl} \mathrm{A}$ respectively. A fuzzy point [7] with a singleton support $\mathrm{x}$ (say) and value $\alpha(0<\alpha \leq 1)$ at $x$ is denoted by $x_{\alpha}$. For a fuzzy set $\mathrm{A}$, the support and complement of $\mathrm{A}$ are denoted by $A_{o}$ and $A^{\prime}$ (or $1-A$ ) respectively. For a fuzzy point $x_{\alpha}$ and a fuzzy set $A$, we write $x_{\alpha} \in A$ iff $\alpha \leq A(x)$, and $x_{\alpha}$ is said to be quasi-coincident (q-coincident, for short) with $\mathrm{A}$, denoted by $x_{\alpha} q A$, iff $\alpha>A^{\prime}(x)$. $A$ is said to be a q-neighbourhood (q-nbd, for short) of $x_{\alpha}$ iff there exists a fuzzy open set $B$ such that $x_{\alpha} q B \leq A$. For two fuzzy sets $A$ and $B, A \leq B$ iff $A B^{\prime}$, and a fuzzy point $x_{\alpha} \in$ $\mathrm{Cl} A$ iff each q-nbd of $x_{\alpha}$ is q-coincident with $\mathrm{A}$ [7]. For the definitions of fuzzy regularly open, regularly closed, semi-open and semi-closed sets we refer to Azad [10]. Simply by X and Y we shall mean the fuzzy topological spaces $(X, T)$ and $\left(Y, T_{1}\right)$ respectively. The constant fuzzy sets $0_{X}$ and $1_{X}$ are defined by $O_{X}(y)=0$ and $1_{X}(y)=1$, for each $y \in X$.

2. FUZZY $\Theta$-CLOSURE AND ITS PROPERTIES.

DEFINITION 2.1. A fuzzy point $x_{\alpha}$ is said to be a fuzzy $\Theta$-cluster point ( $\delta$-cluster point [13]) of a fuzzy set A iff closures of every open q-nbd (resp. iff every regularly open q-nbd) of $x_{\alpha}$ is $q$ coincident with A.

The union of all fuzzy $\Theta$-cluster ( $\delta$-cluster) points of $A$ is called the fuzzy $\Theta$-closure of $A$ and is denoted by $[A]_{\Theta}$ (resp. $[A]_{\delta}$ ). A fuzzy set $A$ will be called fuzzy $\Theta$-closed ( $\delta$-closed) iff $A=[A]_{\Theta}$ (resp. $\left.A=[A]_{\delta}\right)$. It is known [6] that for any fuzzy set $\mathrm{A}$ in an fts $X, \mathrm{Cl} A \leq[A]_{\delta} \leq[A]_{\Theta}$, but the reverse implications are false (see [6] and [13]). However, it is true (see [6]) that for a fuzzy open set $A$ in an fts $X, \mathrm{Cl} A=[A]_{\delta}=[A]_{\Theta}$.

THEOREM 2.2. In an fts $(X, T)$, the following hold:

(a) Finite union and arbitrary intersection of $\Theta$-closed sets in $X$ is fuzzy $\Theta$-closed.

(b) For two fuzzy sets $A$ and $B$ in $X$, if $A \leq B$ then $[A]_{\Theta} \leq[B]_{\Theta}$.

(c) The fuzzy sets $0_{X}$ and $1_{X}$ are fuzzy $\Theta$-closed.

PROOF. The straightforward proofs are omitted.

REMARK 2.3. The complements of fuzzy $\Theta$-closed sets in an $\mathrm{fts}(X, T)$ induce a fuzzy topology $T_{\Theta}$ (say) which is coarser than the fuzzy topology $T$ of the space. Again, for a fuzzy set $A$ in $X$, $[A]_{\Theta}$ is evidently fuzzy closed but not necessarily fuzzy $\Theta$-closed as is seen from the next example. Thus, fuzzy $\Theta$-closure operator is not a Kuratowski closure operator. However, it will be shown in the next section that for any fuzzy set $A$ in an fts $X,[A]_{\Theta}$ is fuzzy $\Theta$-closed if the space $X$ is fuzzy regular (see Corollary 3.6), or iff the space $X$ is fuzzy almost regular (see Theorem 3.10).

EXAMPLE 2.4. Let $X=\{a, b, c\}$ and $T=\left\{0_{X}, 1_{X}, A, B\right\}$, where

and

$$
A(a)=0.5, A(b)=0.6, A(c)=0.2
$$

$$
B(a)=0.4, B(b)=0.5, B(c)=0.1 \text {. }
$$

Let $\mathrm{U}$ be any fuzzy set given by, $U(a)=U(b)=0.3$ and $U(c)=0$. Then, $a_{.6} \in[U]_{\Theta}, a_{.8} \notin[U]_{\Theta}$, but $a_{.8} \in\left[a_{.6}\right]_{\Theta} \leq\left[[U]_{\Theta}\right]_{\Theta}$. Thus, $[U]_{\Theta} \neq\left[[U]_{\Theta}\right]_{\Theta}$. Hence, $[U]_{\Theta}$ is not fuzzy $\Theta$-closed.

In the following example, we observe a deviation from the corresponding established result [ 3 ] in general topology that $x \in[y]_{\Theta}$ iff $y \in[x]_{\Theta}$, if $x, y$ are two points in a topological space. 
EXAMPLE 2.5. Let $X$ be an ordinary set with at least two distinct points $a, b$. Consider the fuzzy topology $T=\left\{0_{X}, 1_{X} A\right\}$, where $A(a)=\frac{1}{2}, A(b)=\frac{2}{5}$ and $A(x)=0$, for $x \neq a, b(x \in X)$. Let us consider the fuzzy points $a_{\frac{1}{12}}$ and $b_{\frac{4}{5}}$. It can be checked that $a_{\frac{1}{12}} \in\left[b_{\frac{4}{5}}\right]_{\Theta}$, but $b_{\frac{4}{5}} \notin\left[a_{\frac{1}{12}}\right]_{\Theta}$,

THEOREM 2.6. For any fuzzy set $A$ in an fts $(X, T),[A]_{\Theta}=\cap\left\{[U]_{\Theta}: U \in T\right.$ and $\left.A \leq U\right\}$.

PROOF. Obviously, L.H.S. $\leq$ R.H.S. Now, if possible let $x_{\alpha} \in$ R.H.S. but $x_{\alpha} \notin[A]_{\Theta}$. Then there exists an open q-nbd $V$ of $x_{\alpha}$ such that $\mathrm{Cl} \not A$ and hence $A \leq 1-C 1 V$. Then $x_{\alpha} \in[1-C 1 V]_{\Theta}$ and consequently, $\mathrm{Cl} V q(1-C 1 V)$ which is impossible.

According to $\mathrm{Pu}$ and Liu [7] a function $S: D \rightarrow J$ is called a fuzzy net in $X$, where $(D, \geq)$ is a directed set and $\mathrm{J}$ denote the collection of all fuzzy points in X. It is denoted by $\left\{S_{n}, n \in D\right\}$ or simply by $(S, D)$. We now set the following:

DEFINITION 2.7. Let $\left\{S_{n}, n \in D\right\}$ be a fuzzy net and $x_{\alpha}$ a fuzzy point in $X$.

(a) $x_{\alpha}$ is called a $\Theta$-cluster point of the fuzzy net iff for every open q-nbd $W$ of $x_{\alpha}$ and for any $n \in D$, there exists $m \geq n(m \in D)$ such that $S_{m} q C l W$.

(b) The fuzzy net is said to be $\Theta$-converge to $x_{\alpha}$ if for any open q-nbd $U$ of $x_{\alpha}$, exists $m \in D$ such that $S_{n} q C 1 U$, for all $n \geq m(n \in D)$. This is denoted by $S \stackrel{\Theta}{\rightarrow} x_{\alpha}$.

THEOREM 2.8. A fuzzy point $x_{\alpha}$ is a $\Theta$-cluster point of a fuzzy net $\left\{s_{n}, n \in D\right\}$ in $X$ iff there is a subnet of $\left\{S_{n}, n \in D\right\}$, which $\Theta$-converges to $x_{\alpha}$.

PROOF. Let $x_{\alpha}$ be a $\Theta$-cluster point of the given fuzzy net. Let $Q x_{\alpha}$ denote the set of closures of all open q-nbds of $x_{\alpha}$. Now for any member $A$ of $Q x_{\alpha}$, there exists an element $S_{n}$ of the net such that $S_{n} q A$. Let $E$ denote the set of all ordered pairs $(n, A)$ with the above property, i.e., $n \in D, A \in Q x_{\alpha}$ and $S_{n} q A$. Then $(E, \gg)$ is a directed set, where $(m, A) \gg(n, B),((m, A),(n, B) \in E)$ iff $m \geq n$ in $D$ and $A \leq B$. Then $T:(E, \gg) \rightarrow(X, T)$ given by $T(m, A)=S_{m}$ can be checked by a subnet of $\left\{S_{n}, n \in D\right\}$. To show that $T \stackrel{\Theta}{\rightarrow} x_{\alpha}$, let $V$ be any open q-nbd of $x_{\alpha}$. Then there exists $n \in D$ such that $(n: C 1 V) \in E$ and then $S_{n} q C l V$. Now, for any $(m, A) \gg(n, C 1 V), T(m, A)=S_{m} q A \leq C 1 V$. Hence, $T \stackrel{\Theta}{\oplus} x_{\alpha}$. Converse is clear.

THEOREM 2.9. Let $A$ be a fuzzy set in $X$. A fuzzy point $x_{\alpha} \in[A]_{\Theta}$ iff there exists a fuzzy net in $A, \Theta$-converging to $x_{\alpha}$.

PROOF. Let $x_{\alpha} \in[A]_{\Theta}$. For each open q-nbd $U$ of $x_{\alpha}, C 1 U q A$. That is, there exist $y^{U} \in A_{o}$ and real number $\beta_{U}$ with $0<\beta_{U} \leq A\left(y^{U}\right)$ such that $y_{\beta}^{U} \in A$ and $y_{\beta}^{U}{ }_{q} C 1 U$. We choose and fix one such $y_{\beta}^{U}$ for each $U$. Let $D$ denote the set of all open q-nbds of $x_{\alpha}$. Then $(D, \geq)$ is directed under inclusion relation, i.e., for $B, C \in D, B \geq C$ iff $B \leq C$. Then $\left\{y_{\beta}^{U} \in A: y_{\beta}^{U} q C 1 U\right.$ and $\left.U \in D\right\}$ is a fuzzy net in $A$ such that it $\Theta$-converges to $x_{\alpha}$. Converse is straightforward even if $x_{\alpha}$ is a $\Theta$-cluster point of the fuzzy net in $A$.

3. CHARACTERIZATIONS OF CERTAIN SEPARATION AXIOMS AND FUNCTIONS IN TERMS OF FUZZY $\Theta$-CLOSURE AND $\delta$-CLOSURE.

DEFINITION 3.1. [9] An fts $X$ is called fuzzy strongly $T_{2}$ iff for any two distinct fuzzy points $x_{\alpha}$ and $y_{\beta}$ in $X$ : whenever $x \neq y, x_{\alpha}$ and $y_{\beta}$ have fuzzy open nbds $U$ and $V$ respectively such that $U q V$; and when $x=y, \alpha<\beta$ (say), there exist fuzzy open sets $U$ and $V$ such that $x_{\alpha} \in U, y_{\beta} q V$ and $U \phi V$.

LEMMA 3.2. For any two fuzzy open sets $A$ and $B$ in an fts $(X, T), A q B \Rightarrow C 1 A q B$ and $A \phi C 1 B$.

THEOREM 3.3. An fts $(X, T)$ is fuzzy strongly $T_{2}$ iff every fuzzy point of $X$ is fuzzy $\Theta$-closed, and for $x, y \in X$ with $x \neq(C 1 U)_{o}$.

PROOF. Let $X$ be fuzzy strongly $T_{2}$, and let $x_{\alpha}$ be a fuzzy point in $X$. In order to show that $\left[x_{\alpha}\right]_{\Theta}=x_{\alpha}$, it suffices to establish that for any fuzzy point $y_{\beta}, y_{\beta} \notin\left[x_{\alpha}\right]_{\Theta}$ when either $x \neq y$, or $x=y$ and $\beta>\alpha$. In the first case, there exist fuzzy open nbds $U$ and $V$ of $y_{1}$ and $x_{\alpha}$ respectively such that $U \phi V$ and then $C 1 U \phi V$ (by Lemma 3.2). Then $U$ is an open q-nbd of $y_{\beta}$ with $C 1 U \phi x_{\alpha}$ so that $Y_{\beta} \notin\left[x_{\alpha}\right]_{\Theta}$. In the second case, there exist a fuzzy open nbd $U$ of $x_{\alpha}$ and an open q-nbd $V$ of $y_{\beta}$ such that $U q V$. Then $C 1 V q U$ so that $C 1 V \phi x_{\alpha}$ and hence $y_{\beta} \notin\left[x_{\alpha}\right]_{\Theta}$. Finally, for two distinct points $x, y$ of $X$, there exist fuzzy open nbds $U$ of $x_{1}$ and $V$ of $y_{1}$ such that $U q V$ and hence $C 1 U q V$, i.e., 
$y_{1} \in V \leq 1-C 1 U$. Then $(1-C 1 U)(y)=1 \Rightarrow(C 1 U)(y)=0 \Rightarrow y \notin(C 1 U)_{0}$. Conversely, let $x_{\alpha}$ and $y_{\beta}$ be two distinct fuzzy points in $X$.

CASE I. Let $x \neq y$. First suppose that at least one of $\alpha$ and $\beta$ is less than 1 , say $\alpha<1$. Then there exists $\lambda>0$ such that $\alpha+\lambda<1$. Now $x_{\lambda} \notin\left[y_{\beta}\right]_{\Theta}$ and hence there exists a fuzzy open nbd $U$ of $y_{\beta}$ such that $x_{\lambda} \notin[U]_{\Theta}$ (by Theorem 2.6). Then $U_{\ell C 1 V}$, for an open q-nbd $V$ of $x_{\lambda}$. Since $V(x)>1-\lambda>\alpha, V$ and $U$ are fuzzy open nbds of $x_{\alpha}$ and $y_{\beta}$ respectively such that $U \alpha V$.

Next, suppose $\alpha-\beta-1$. By hypothesis, there exists a fuzzy open nbd $U$ of $x_{1}$ such that $(C 1 U)(y)=0$. Then $(1-C 1 U)$ is a fuzzy open nbd of $y_{1}$ such that $U(1-C 1 U)$.

CASE II. Let $x=y$. Suppose $\alpha<\beta$. Then $y_{\beta} \notin\left[s_{\alpha}\right]_{\Theta}$ and so $y_{\beta} \notin[U]_{\Theta}$, for some fuzzy open nbd $U$ of $x_{\alpha}$. Then for an open q-nbd $V$ of $y_{\beta}, C 1 V \phi U$ and hence $V \phi U$.

DEFINITION 3.4. [6] An fts $X$ is said to be:

(a) fuzzy regular (semi-regular) iff for each fuzzy point $x_{\alpha}$ in $X$ and each open q-nbd $U$ of $x_{\alpha}$, there exists an open q-nbd $V$ of $x_{\alpha}$ such that $C 1 V \leq U$ (resp. Int $C 1 V \leq U$ );

(B) fuzzy almost regular iff for each fuzzy point $x_{\alpha}$ in $X$ and each regularly open q-nbd $U$ of $x_{\alpha}$, there exists a regularly open $\mathrm{q}$-nbd $V$ of $x_{\alpha}$ such that $C 1 V \leq U$.

THEOREM 3.5. An fts $X$ is:

(a) fuzzy regular iff for any fuzzy set $A$ in $X, C 1 A=[A]_{\Theta}$;

(b) fuzzy semi-regular iff $[A]_{\delta}=C 1 A$, for any fuzzy set $A$ in $X$.

PROOF. Let $X$ be fuzzy regular. For any fuzzy set $A$ in $X$ it is always true that $C 1 A \leq[A]_{\Theta}$. Now, let $x_{\alpha}$ be a fuzzy point in $X$ such that $x_{\alpha} \in[A]_{\Theta}$ and let $U$ be any open q-nbd of $x_{\alpha}$. Then by fuzzy regularity of $X$, there exists an open q-nbd $V$ of $x_{\alpha}$ such that $C 1 V \leq U$. Now, $x_{\alpha} \in[A]_{\Theta} \Rightarrow C 1 V q A \Rightarrow U q A \Rightarrow x_{\alpha} \in C 1 A$. Thus $[A]_{\Theta}=C 1 A$.

Conversely, let $x_{\alpha}$ be a fuzzy point in $X$ and $U$ an open q-nbd of $x_{\alpha}$. Then $x_{\alpha} \notin(1-U)=C 1(1-U)=[1-U]_{\theta}$. Thus there exists an open q-nbd $V$ of $x_{\alpha}$ such that $C 1 V \phi(1-U)$ and then $C 1 V \leq U$. Hence $X$ is fuzzy regular. (b) Similar to (a) and is omitted.

COROLLARY 3.6. In a fuzzy regular space $(X, T)$, a fuzzy closed set is fuzzy $\Theta$-closed, and hence for any fuzzy set $A$ in $X,[A]_{\Theta}$ is fuzzy $\Theta$-closed.

LEMMA 3.7. For any fuzzy semi-open set $A$ in $X,[A]_{\delta}=C 1 A$.

PROOF. It suffices to show that $[A]_{\delta} \leq C 1 A$. Let $x_{\alpha} \notin C 1 A$. Then there exists an open $\mathrm{q}$-nbd $V$ of $x_{\alpha}$ such that $V q A$. Then IntC1 $V \leq \operatorname{Int} C 1(1-A)=1-C 1 \operatorname{Int} A \leq 1-A$ (since $A$ is fuzzy semiopen). Thus Int $\mathrm{C} 1 V \notin A$ and consequently, $x_{\alpha} \notin[A]_{\delta}$.

THEOREM 3.8. An fts $X$ is fuzzy almost regular iff $[A]_{\Theta}=C 1 A$, for every fuzzy semi-open set $\boldsymbol{A}$ in $\boldsymbol{X}$.

PROOF. Let $X$ be fuzzy almost regular and $A$ any fuzzy semi-open set in $X$. It is enough to show that $[A]_{\Theta} \leq C 1 A$. Suppose $x_{\alpha} \notin C 1 A$. By Lemma 3.7, there exists an open q-nbd $V$ of $x_{\alpha}$ such that Int $C 1 V q A$. Since $X$ is fuzzy almost regular, there is a fuzzy regularly open set $U$ such that $x_{\alpha} U \leq C 1 U \leq$ Int $C 1 V \leq 1-A$. Then $C 1 U \phi A$ and hence $x_{\alpha} \notin[A]_{\Theta}$. Conversely, let $U$ be any fuzzy regularly open q-nbd of a fuzzy point $x_{\alpha}$. Then $x_{\alpha} \notin 1-U=C 1(1-U)=[1-U]_{\Theta}$, since a fuzzy regularly closed set is fuzzy semi-open. Hence, there is an open q-nbd $V$ of $x_{\alpha}$ such that $C 1 V q(1-U)$. Since $V \leq$ Int $C 1 V$, Int $C 1 V$ is a regularly open q-nbd of $x_{\alpha}$ such that $C 1$ Int $C 1 V=C 1 V \leq U$ and $X$ is fuzzy almost regular.

THEOREM 3.9. In an $\mathrm{fts} X$, the following statements are equivalent:

(a) For any fuzzy open set $A$ in $X,\left[[A]_{\Theta}\right]_{\Theta}=[A]_{\Theta}$.

(b) For any fuzzy set $A$ in $X,\left[[A]_{\Theta}\right]_{\Theta}=[A]_{\Theta}$.

(c) For any fuzzy set $A$ in $X,[A]_{\Theta}=[A]_{\delta}$.

(d) $X$ is fuzzy almost regular.

PROOF. (a) $\Rightarrow\left(\right.$ d): We first show that for any fuzzy regularly closed set $F$ in $X, F=[F]_{\Theta}$. In fact, $F$ being fuzzy regularly closed, $F=C 1 U$, for some fuzzy open set $U$. Now, $\left.\left.[F]_{\Theta}=[C 1 U]_{\Theta}\right][U]_{\Theta}\right]_{\Theta}$ (since $U$ is fuzzy open) $=[U]_{\Theta}=C 1 U=F$. Next, let $x_{\alpha}$ be a fuzzy point in $X$ and $A$ any fuzzy 
regularly open set in $X$ with $x_{\alpha} q A$. Then $x_{\alpha} \notin(1-A)=[1-A]_{\Theta}$, since $(1-A)$ is fuzzy regularly closed. Hence, there exist a fuzzy open set $V$ such that $x_{\alpha} q V$, but $C 1 V \phi(1-A)$. Let $W=\operatorname{Int} C 1 \mathrm{~V}$. Then $x_{\alpha} q W$, and $C 1 W=C 1 V q(1-A)$. Thus, $W$ is a regularly open q-nbd of $x_{\alpha}$ such that $C 1 W \leq A$. Hence, $X$ is fuzzy almost regular.

(d) $\Rightarrow$ (c): For any fuzzy set $A$, it is clear that $[A]_{\delta} \leq[A]_{\Theta}$. Now, let $x_{\alpha} \in[A]_{\Theta}$ and $U$ an open qnbd of $x_{\alpha}$. Then $x_{\alpha} q$ Int $C 1 U$. By (d), there exists a regularly open q-nbd $V$ of $x_{\alpha}$ such that $C 1 V \leq$ Int $C 1 U$. Now, $x_{\alpha} \in[A]_{\Theta} \Rightarrow C 1 V q A \Rightarrow \operatorname{Int} C 1 U q A \Rightarrow x_{\alpha} \in[A]_{\delta}$.

(c) $\Rightarrow(\mathrm{b}):\left[[A]_{\Theta}\right]_{\Theta}=\left[[A]_{\delta}\right]_{\Theta}=\left[[A]_{\delta}\right]_{\delta}=[A]_{\delta}=[A]_{\Theta}$.

(b) $\Rightarrow(\mathrm{a})$ : Obvious.

DEFINITION 3.10. A function $f: X \rightarrow Y$ from an fts $(X, T)$ to another $\mathrm{fts}\left(Y, T_{1}\right)$ is called

(i) fuzzy weakly continuous [10] iff for each fuzzy open set $A$ in $Y, f^{-1}(A) \leq \operatorname{Int} f^{-1}(C 1 A)$.

(ii) fuzzy $\Theta$-continuous [6] iff for each fuzzy point $x_{\alpha}$ in $X$ and each open q-nbd $V$ of $x_{\alpha}, f(C 1 U) \leq C 1 V$, for some open q-nbd $U$ of $x_{\alpha}$.

LEMMA 3.11. Let $f: X \rightarrow Y$ be a function. Then for a fuzzy set $B$ in $Y, f\left(1-f^{-1}(B)\right) \leq 1-B$, where equality holds if $f$ is onto.

PROOF. Let $y \in Y$. If $f^{-1}(y)=\varnothing$, then $\left[f\left(1-f^{-1}(B)\right)\right](y)=0 \leq(1-B)(y)$. If $f^{-1}(y) \neq \varnothing$, then $\left[f\left(1-f^{-1}(B)\right)\right](y)=\operatorname{Sup}_{x \in f^{-1}(y)}\left[1-f^{-1}(B)\right](x)=\operatorname{Sup}_{x \in f^{-1}(y)}\{1-B(f(x))\}$

$$
=\operatorname{Sup}_{x \in f^{-1}(y)}\{1-B(y)\}=1-B(y)=(1-B)(y) .
$$

If $f$ is onto, then for each $y \in Y, f^{-1}(y) \neq \varnothing$, and hence we have $f\left(1-f^{-1}(B)\right)=1-B$.

THEOREM 3.12. A function $f: X \rightarrow Y$ is:

(a) fuzzy weakly continuous iff $f(C 1 U) \leq[f(U)]_{\Theta}$, for each fuzzy set $U$ in $X$.

(b) fuzzy $\Theta$-continuous iff $f\left([A]_{\Theta}\right) \leq[f(A)]_{\Theta}$, for any fuzzy set $A$ in $X$.

PROOF. (a) Let $f$ be a fuzzy weakly continuous and $U$ any fuzzy set in $X$. Suppose $x_{\alpha} \in C 1 U$. It is enough to show that $f\left(x_{\alpha}\right) \in[f(U)]_{\Theta}$. Let $A$ be any open q-nbd of $f\left(x_{\alpha}\right)$. Then $f^{-1}(A) q x_{\alpha}$. By fuzzy weak continuity of $f, f^{-1}(A) \leq \operatorname{Int} f^{-1}(C 1 A)$ and hence Int $f^{-1}(C 1 A)$ is an open q-nbd of $x_{\alpha}$. Since $x_{\alpha} \in C 1 U$, we have Int $f^{-1}(C 1 A) q U$. Then $f^{-1}(C 1 A) q U$ and hence $C 1 A q f(U)$. Thus $f\left(x_{\alpha}\right) \in[f(U)]_{\Theta}$.

Conversely, for any fuzzy open set $U$ in $Y, f\left(1-\operatorname{Int} f^{-1}(C 1 U)\right)$

$$
=f\left(C 1\left(1-f^{-1}(C 1 U)\right)\right) \leq\left[f\left(1-f^{-1}(C 1 U)\right]_{\Theta} \leq[1-C 1 U]_{\Theta}\right.
$$

(by Lemma 3.11)

$$
\begin{array}{r}
=C 1(1-C 1 U)=1-\operatorname{Int} C 1 U \leq 1-U \Rightarrow f\left(1-\operatorname{Int} f^{-1}(C 1 U)\right) d U \Rightarrow 1-\operatorname{Int} f^{-1}(C 1 U) d f^{-1}(U) \\
\Rightarrow f^{-1}(U) \leq \operatorname{Int} f^{-1}(C 1 U)
\end{array}
$$

Hence $f$ is fuzzy weakly continuous.

(b) Let the condition hold. For any fuzzy point $x_{\alpha}$ in $X$ and any open q-nbd $A$ of $f\left(x_{\alpha}\right)$ in $Y$, we have by Lemma 3.11, $f\left(1-f^{-1}(C 1 A)\right) \leq 1-C 1 A$. Thus, $C 1 A q f\left(1-f^{-1}(C 1 A)\right)$ so that $f\left(x_{\alpha}\right) \notin\left[f\left(1-f^{-1}(C 1 A)\right)\right]_{\Theta}$. By hypothesis, $f\left(x_{\alpha}\right) \notin f\left(\left[1-f^{-1}(C 1 A)\right]_{\Theta}\right)$ and hence $x_{\alpha} \notin\left[1-f^{-1}(C 1 A)\right]_{\Theta}$. Then there is an open q-nbd $V$ of $x_{\alpha}$ such that $C 1 V q\left(1-f^{-1}(C 1 A)\right)$ and hence $f(C 1 V) \leq f f^{-1}(C 1 A) \leq C 1 A$. Thus $f$ is fuzzy $\Theta$-continuous.

The converse part was proved in [6].

\section{REFERENCES}

1. VELIČKO, N.V. H-closed topological spaces, Trans. Amer. Math. Soc. 78(1968), 103-118.

2. DICKMAN, R.F. JR., and PORTER, J.R. $\Theta$-closed subsets of Hausdorff spaces, Pacific J. Math. 58(1975), 407-415.

3. ESPELIE, M.S., and JOSEPH, J.E. Some properties of $\Theta$-closure, Canad. J. Math. 33(1981), 142-149. 
4. SIVARAJ, D. Semi-open set characterizations of almost regular spaces, Glasnik Math. 21 (1986), 437-440.

5. CHANG, C.L. Fuzzy topological spaces, J. Math. Anal. Appl. 24(1968), 182-190.

6. MUKHERJEE, M.N., and SINHA, S.P. On some near-fuzzy continuous functions between fuzzy topological spaces, Fuzzy Sets and Systems 34(1990), 245-254.

7. PU, P.M. and LIU, Y.M. Fuzzy topology I. Neighbourhood structure of a fuzzy point and Moore-Smith convergence, J. Math. Anal. Appl. 76(1980), 571-599.

8. PU, P.M. and LIU, Y.M. Fuzzy topology II. produce and quotient spaces, J. Math. Anal. Appl. 77(1980), 20-37.

9. GANGULY, S. and SAHA, S. On separation axioms and $T_{1}$ fuzzy continuity, Fuzzy Sets and Systems 16(1985), 265-275.

10. AZAD, K.K. On fuzzy semicontinuity, fuzzy almost continuity and fuzzy weakly continuity, J. Math. Anal. Appl. 82(1981), 14-32.

11. MUKHERJEE, M.N. and SINHA, S.P. On some weaker forms of fuzzy continuous and fuzzy almost open functions on fuzzy topological spaces, Fuzzy Sets and Systems 32(1989), 103-114.

12. ZADEH, L.A. Fuzzy sets, Inform. Control 8(1965), 338-353.

13. GANGULY, S. and SAHA, S. A note on $\delta$-continuity and $\delta$-connected sets in fuzzy set theory, Simon Stevin 62(1988), 127-141. 


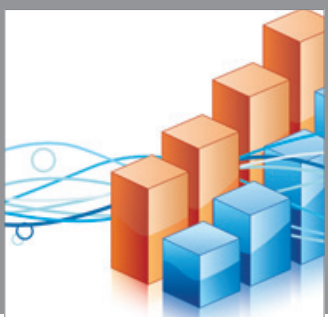

Advances in

Operations Research

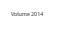

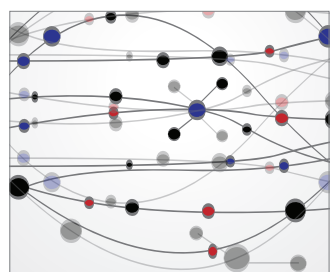

\section{The Scientific} World Journal
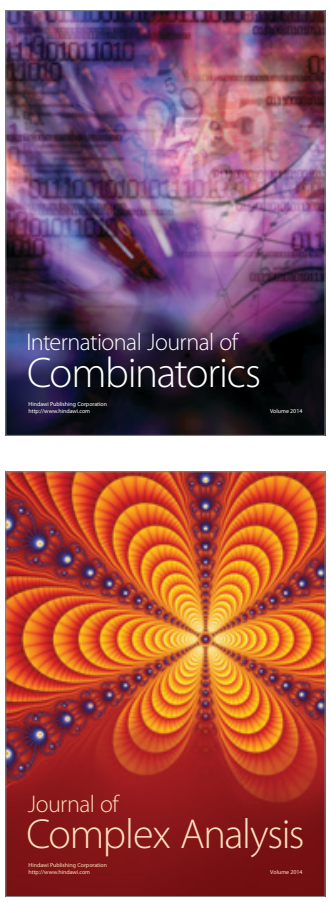

International Journal of

Mathematics and

Mathematical

Sciences
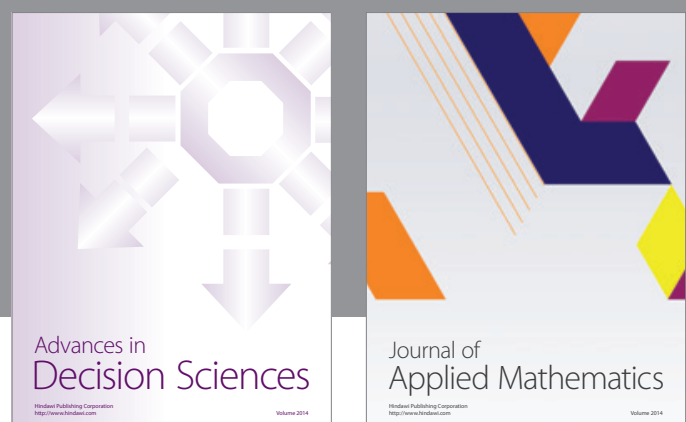

Journal of

Applied Mathematics
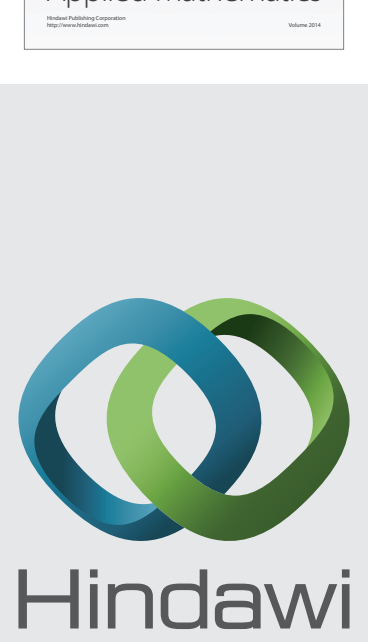

Submit your manuscripts at http://www.hindawi.com
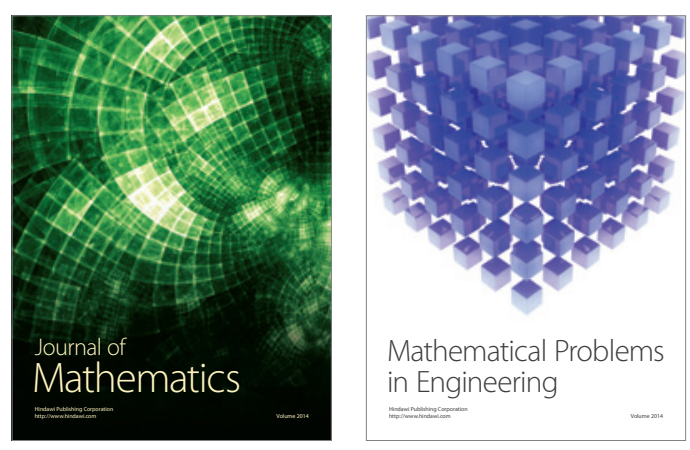

Mathematical Problems in Engineering
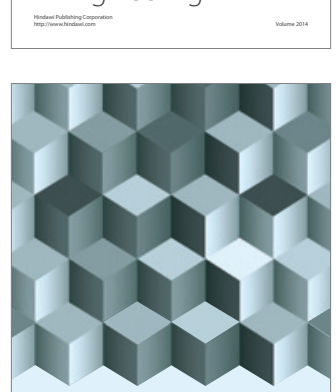

Journal of

Function Spaces
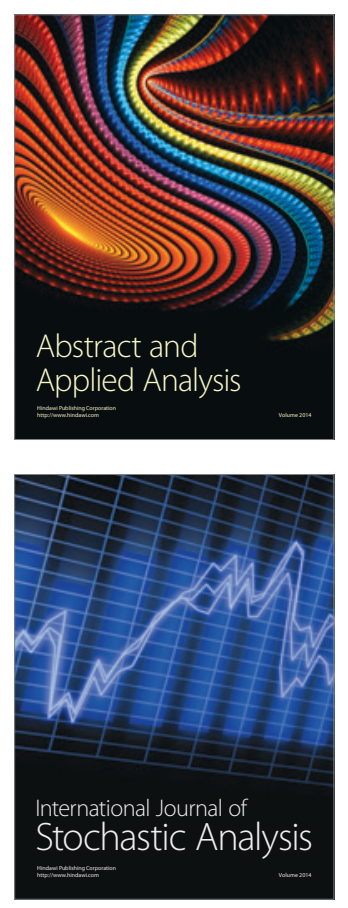

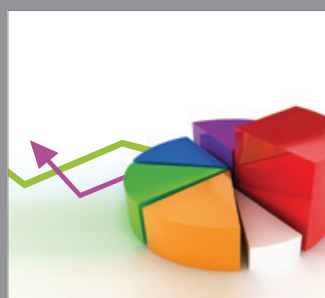

ournal of

Probability and Statistics

Promensencen
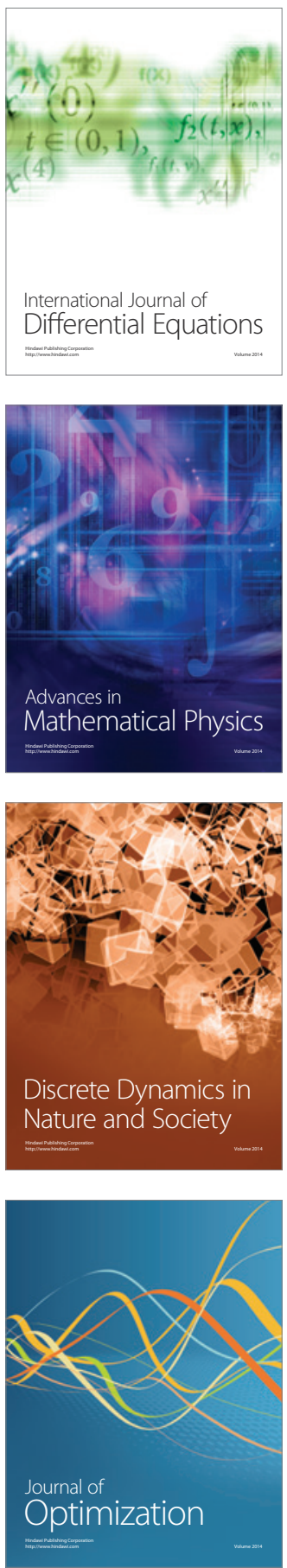